\title{
WHAT DETERMINES THE BULGE TO DISK RATIO OF GALAXIES
}

\author{
Nico Roos \\ Sterrewacht Leiden \\ Leiden, the Netherlands
}

\begin{abstract}
Galaxies having the same luminosity may have very different bulge to disk ratios, while the mean bulge to disk ratio slowly increases with total luminosity (Schechter and Dressler, 1987, Sandage et al., 1985). Such a behaviour is expected if ellipticals and the spheroidal components of disk galaxies are produced by secondary accretion of galaxies by larger galaxies. This is illustrated using a simple toy model of the evolution of the mass function of galaxies due to galaxy mergers.
\end{abstract}

\section{Introduction}

The form of the galaxy luminosity function (all types included) is not sensitive to the environment. However, the fraction of early type galaxies increases slowly with local galaxy density (Dressler, 1980, Postman and Geller, 1984). The increase in the fraction of early-type galaxies occurs predominantly at the high luminosity end of the luminosity function (Schechter and Dressler, 1987, Sandage et al., 1985). These are important clues to our understanding of the origin of Hubble sequence of galaxies. Another, perhaps equally important clue is that galaxies having similar luminosities and similar environments can have very different bulge to disk ratios (Schechter and Dressler, 1987; see figure 1). These facts taken together suggest that some stochastic process acting preferentially in regions of higher galaxy density, and affecting predominantly the more massive galaxies, has transformed some fraction of (originally late-type) galaxies into early-type galaxies. Galaxy interactions and mergers are of course prime candidates for this process.

Galaxy mergers are a natural part of the hierarchical clustering process in bottom-up scenarios for the formation of galaxies and clusters such as the Cold Dark Matter cosmology (Blumenthal et al. 1984). Luminous galaxies are supposed to form dissipatively in the potential wells of the dark matter. They may then survive as rapidly rotating and flattened subunits during the subsequent formation of groups and clusters (White and Rees,1978, Carlberg and Couchman, 1989). In such a scenario it is plausible that the formation and evolution of galaxies has evolved from a relatively rapid, dissipational collapse of mainly gaseous matter, towards slow accretion of more clumpy, mainly stellar systems. It seems plausible that galaxies were formed as gaseous disk-like systems at a redshift of 2 to 3 (Wolfe, 1989). Star formation in these sytems may have taken a much longer time and may have occurred in bursts (Lilly and Longair, 1984, Djorgovski, 1987), perhaps triggered by accretion events. One of the important questions is then: did bright elliptical galaxies and the spheroidal components of disk galaxies form early, when galaxies were still mainly 
gaseous (Silk and Norman, 1981), or did they form later? One way of obtaining an answer is to study the effects of galaxy interactions and mergers at the present epoch and try to make an extrapolation into the past.

This approach lead Toomre and Toomre (1972, see also Toomre 1977) to suggest that (bright) elliptical galaxies were the remnants of past mergers between spiral galaxies. This suggestion gained support from numerical studies of mergers in collapsing groups and clusters (Roos and Norman, 1979, Roos and Aarseth, 1982) and from cosmological simulations of galaxy clustering (Aarseth and Fall, 1979, Jones and Efstathiou, 1979, Roos, 1981). Several objections were raised against this hypothesis (Ostriker, 1980, Tremaine 1981, van den Bergh, 1984, Carlberg, 1986). The most important ones are probably: (i) can the high phase space density in the central regions of elliptical galaxies be explained without invoking extra dissipation (see Lake, 1989), and (ii) why do ellipticals have more globular clusters per unit luminosity than disk galaxies. In the last few years the evidence in favour of the merger hypothesis has grown considarably by both observational and theoretical studies of merger remnants (Schweizer, 1986, Lake and Dressler, 1986, Barnes, 1988), by the discovery of shells and ripples around $20 \%$ of ellipticals (Malin and Carter, 1983) and by studies of the kinematics of ellipticals (Franx and Illingworth, 1988).

Most mergers have occurred between galaxies of unequal mass. In a previous paper (Roos, 1981, hereafter Paper I) it was therefore hypothesized that infall of smaller galaxies would contribute to the formation of a galactic bulges along with thickening (and ultimately destruction) of a stellar disk and depletion of gas, leading to evolution of galaxies along the sequence $(\mathrm{Sc} \rightarrow \mathrm{Sb} \rightarrow \mathrm{Sa}) \rightarrow \mathrm{SO} \rightarrow \mathrm{E}$. The most recent estimate of the present merging rate among galaxies indicates that a typical galaxy with luminosity $\mathrm{L}^{*}$, at the break of the galaxy luminosity function has accreted about $0.3 \mathrm{~L}^{*}$ since a redshift $z=0.75$ (Bahcall and Tremaine, 1988), enough to account for a typical bulge mass. Some aspects of this hypothesis such as the morphology - density relation were investigated in Paper I. It was also found that the luminosity function should depend on galaxy type, the fraction of early type galaxies increasing with luminosity. This prediction is confirmed by the work of Sandage et al. (1985). The large scatter in bulge to disk ratios of galaxies having similar total luminosity provides a new argument in favour of the merger hypothesis for the origin of the spheroidal components of galaxies. This is illustrated in the next section using the simple Monte Carlo model of Paper I. A more extensive discussion of the distribution of bulge to disk ratios of galaxies and the dependence of the luminosity function on galaxy type will be published elsewhere.

\section{A toy model for spheroid growth}

As in paper I we assume that initially all the galactic mass is in disks. with a mass function given by the Schechter form:

$$
n(M) d M \propto M^{\alpha} \exp \left(M / M^{*}\right)
$$

where $\alpha=-1.25$. We further assume that the mass to light ratio does not depend on mass. The evolution of this distribution due to merging is then simulated numerically by choosing pairs $\left(M_{1}, M_{2}\right)$ with a probability proportional to $\left(M_{1}+M_{2}\right)^{\lambda}$. The bulge to 

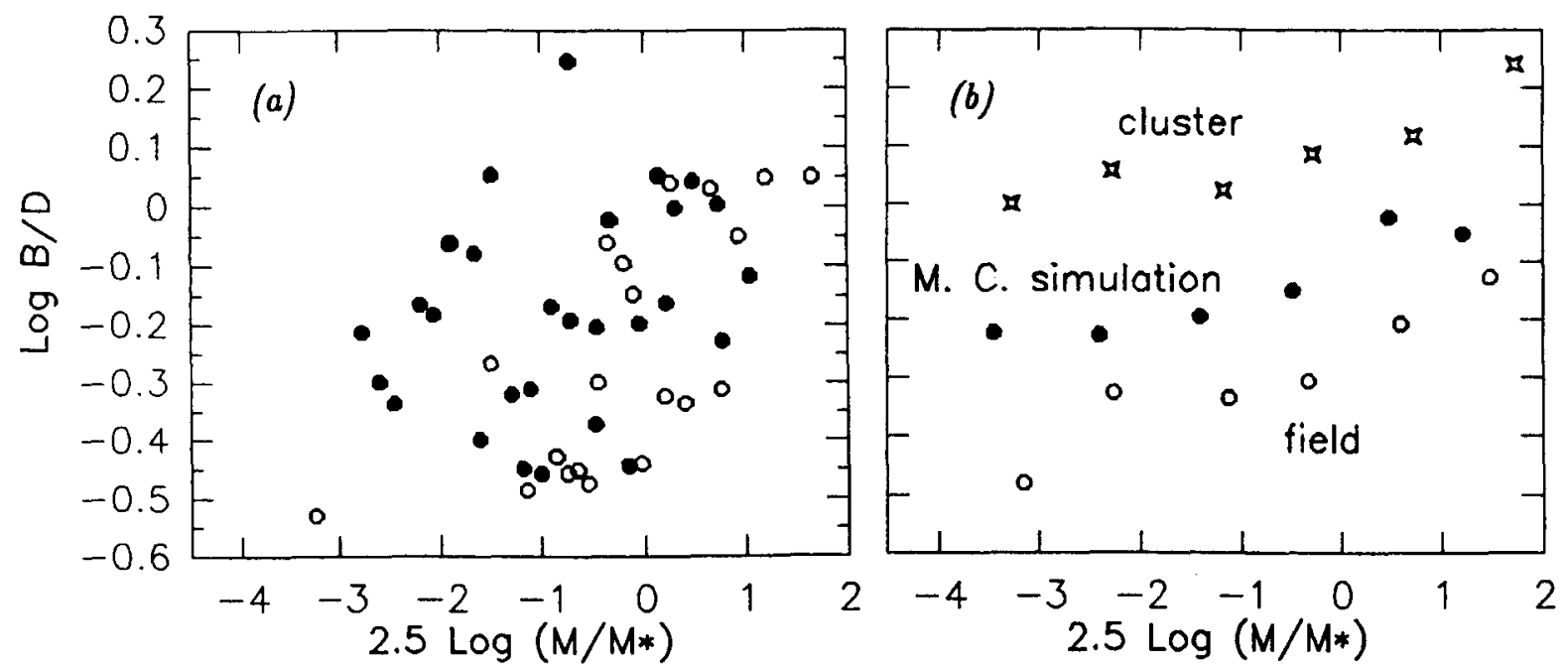

Figure 1 (a) Average bulge to disk ratios as a function of mass (assuming constant $M / L$ ) for Schechter and Dressler's sample of field galaxies (open dots) having apparent visual magnitude brighter than 16.5. Each point represents an average for 10 galaxies having similar luminosities. The filled symbols are results from the toy model discussed below $(\lambda=0)$. (b) Idem, but here the average is determined over all galaxies in a particular magnitude interval. The open stars are mean bulge to disk ratios for Dressler's sample of cluster galaxies (see Schechter and Dressler, 1987)

disk ratio of a galaxy is thought to be represented by $\Delta$, the ratio of total accreted mass over the original mass of the galaxy. The dynamical friction time of a satellite orbiting in a larger (spherical) galaxy is roughly comparable with the orbital time divided by the mass ratio (satellite mass over the mass of the galaxy inside the orbital radius of the satellite) of the two systems. The satellite is tidally stripped as it moves inward. The mass ratio may increase or decrease with separation depending whether the density distribution in the parent galaxy is steeper or shallower than the density distribution of the satellite. So, satellites must be sufficiently massive and sufficiently centrally concentrated in order to have contributed to the mass of the central spheroid in the last $10 \mathrm{Gyr}$. Note that the dominant contribution to $\Delta$ comes from the more massive galaxies for $\lambda>-0.75$. The total number of mergers determines the mean $\Delta$, which may be used as a scaling parameter to be compared with the mean B/D of galaxies. This parameter is a slowly increasing function of local galaxy density (see Paper I, and Roos and Aarseth, 1982). The results for $\lambda=0$ are shown in Figure 1. Figure 1a illustrates the large (intrinsic) scatter in the observed values of $B / D$. The distribution of $\Delta$ in the model is similar to the observed distribution of bulge to disk ratios. The mean $\Delta$ increases slowly as a function of mass (figure 1b). The vertical position of this relation is determined by the total number of mergers, while its slope is determined by the value of $\lambda$.

In the Press and Schechter theory for hierarchical clustering (Press and Schechter, 1974) the slope of the low mass end of the mass function is determined by the index $n$ of the initial density fluctuation spectrum: $\alpha_{P S}=(n-9) / 6$. The Monte Carlo integration of the coagulation equation presented here also leads to a self-similar power law mass function 
with an exponential cut-off. In our case $\alpha_{\text {coag }} \approx-3 \lambda / 2$ for $0<\lambda \leq 1$ (Nakano, 1966, see also Silk and White, 1978). In the cosmological simulations of paper I it was indeed found that the merging probability (and the amplitude of the two-point correlation function) were proportional to mass for an initially random distribution $(n=0)$ of particles. It may thus be possible to give a self-consistent description of the evolution of the mass function of galaxies and clusters which is based on the coagulation formalism.

\section{Discussion}

There is now considerable evidence that ellipticals are the remnants of past mergers. Ellipticals and bulges have similar properties ( Davies, 1987, Kormendy and Djorgovski, 1989). It is therefore likely that they have a similar origin. Photometric and kinematic studies of galaxies with peanut-, X- or box-shaped bulges (Whitmore and Bell, 1988, Binney and Petrou, 1985, Statler, 1988) and of ripples in disk galaxies (Schweizer and Seitzer, 1988, Wallin and Struck-Marcell, 1988) lend support to the merger hypothesis for the origin of spheroidal components of galaxies. Quinn and Goodman (1986, see also Hernquist and Quinn, 1988) have performed numerical simulations of satellite accretion by stellar disks showing that stellar disks are puffed up considerably by a satellite only one tenth the mass of the parent galaxy. This seems to require that stellar disks are (re)formed after such an accretion event. Freeman (1987) has drawn attention to connection between thick disks and bulges. He also proposes that matter stripped from sinking satellites has contributed to the halo population and the thick disk of the Milky Way. Observational evidence for such accretion events during the lifetime of the Milky Way is presented by Searle and Zinn (1978) and Rodgers and Paltaglou (1984).

The main purpose of this contribution is to draw attention to the observational fact that galaxies having similar luminosities and similar environments may have very different bulge to disk ratios. this property is not easily explained in theories in which galaxy formation is regarded as a monolithic collapse of a single gas cloud (Eggen et al., 1962), while it is readily explained as a result of secondary accretion of smaller galaxies. The observed increase of the fraction of early-type galaxies with luminosity and with local

galaxy density are also expected in this model. Further investigations of the evolution of galaxies due to accretion of smaller galaxies may prove to be very fruitful.

\section{References}

Aarseth, S. J., and Fall, S. M. 1980, Ap. J. 236, 43.

Bahcall, S. R. and Tremaine, S. 1988, Ap. J. Letters 326, L1

Barnes, J. E. 1988, Ap. J. 331, 699.

Binney, J, and Petrou, M. 1985,M.N.R.A.S. 214, 449.

Blumenthal, G. R., Faber, S. M., Primack, J. R., and Rees, M. J. 1984, Nature 311, 517.

Carlberg, R. G. 1986, Ap. J. 310, 593.

Carlberg, R. G. and Couchman, H. M. P. 1989, Ap. J. 340, 47.

Davies, 1987, in IAU Symposium bf 127 Structure and Dynamics of Elliptical Galaxies, ed. T. de Zeeuw (Dordrecht: Reidel), p 63. 
Djorgovski, S. 1987, in Starbursts and Galaxy Evolution, ed. T. X. Thuan et al. (Moriond, Edition Frontières) $\mathrm{p} 401$.

Dressler, A. 1980, Ap. J. $236,351$.

Eggen, O., Lynden-Bell, D., Sandage, A. 1962, Ap.J. 136, 748.

Franx, M. and Illingworth, G. D. 1988, Ap. J. Letters 327, L55.

Freeman, K. 1987, Ann. Rev. Astr. Ap. 25, 603.

Hernquist, L., and Quinn, P. J. 1989, in The epoch of Galaxy Formation, eds. C. S. Frenk et al. (Dordrecht: Kluwer Academic Publishers), p 427.

Jones, B. J. T., and Efstathiou, G. 1979 M. N. R. A. S. 189, 27.

Kormendy, J., and Djorgovski, S. 1989, Ann. Rev. Astr. Ap. 27, .

Lake, G., and Dressler, A. 1986, Ap. J. 310, 605.

Lake, G. 1989, Astron. J. 95, 1312.

Lilly, S. J., and Longair, M. S. 1984, M. N. R. A. S. $211,833$.

Malin, D. F., and Carter, D. 1983, Ap. J. 274, 534.

Nakano, T. 1966, Progr. Theor. Phys. 36, 515.

Ostriker, J. P. 1980, Comments on Astrophysics Vol. 8, 177.

Postman, M. and Geller, M. J. 1984, Ap. J. $281,95$.

Press, W. H., and Schechter, P. 1974, Ap. J. 187, 425.

Quinn, P. J., and Goodman, J. 1986, it Ap. J. 309, 372.

Rodgers, A., and Paltaglou, G. 1984, Ap. J. (Letters) 283, L5.

Roos, N., and Norman, C. A. 1979, Astr. Ap. 76, 75.

Roos, N. 1981, (Paper I), Astr. Ap. 95, 349.

Roos, N., and Aarseth, S. J. 1982, Astr. Ap. 114, 42.

Sandage, A. Binggeli, B., and Tammann, G. A. 1985, Astron. J. 90, 1759.

Schechter, P. L., and Dressler, A. 1987, Astron. J. 94, 563.

Schweizer, F. 1986, Science 231, 227.

Schweizer, F., and Seitzer, P. 1988, Ap. J. 328, 88.

Searle, L., and Zinn, R. J. 1978, Ap. J 225, 357.

Silk, J., and Norman, C.A. 1981, Ap. J. 247, 59.

Silk, J., and White, S. D. M. 1978, Ap. J. Letters 223, L59.

Statler, T. S. 1988, Ap. J. 33171.

Toomre, J., and Toomre, A. 1972, Ap. J. 178, 623.

Toomre, J. 1977, in The Evolution of Galaxies and Stellar Populations, eds. B. Tinsley and R. Larson (Yale University Press), p 420.

Tremaine, S. 1981, in The Structure of Normal Galaxies, ed. S. M. Fall and D. Lynden-Bell (Cambridge: Cambridge University Press), p 67.

van den Bergh, S. 1984, in Clusters and Groups of Galaxies, eds. F. Mardirossian et al. (Dordrecht: Reidel), p 139.

Wallin, J. F., and Struck-Marcell, C. 1988, Astron. J. 96, 1850.

White, S. D. M., and Rees, M. J. 1978, M. N. R. A. S. 183, 341.

Whitmore, B. C. and Bell, M. 1988, Ap. J. 324, 74.

Wolfe, A. M. 1989, in The Epoch of Galaxy Formation, eds. C.S. Frenk et al. (Dordrecht: Kluwer Academic Publishers), p 101. 


\section{DISCUSSION}

Whitmore: If bulges are produced by merging with smaller mass galaxies we would expect that roughly 508 are counter rotating with respect to the disk. I am not aware of any such cases. Are you?

Roos: No, I am not, but I am not sure we should expect that. I expect that big bulges indeed are very similar to elliptical galaxies. They might also show peculiar kinematics as found in many ellipiticals. Small bulges or spheroids, however, may be formed by small nearby satellite galaxies. I could imagine that in the tidal torque model for the origin of angular momentum of disk galaxies the orbital angular momentum of nearby small companions is correlated with the angular momentum of the big galaxy.

Navarro: How do thin disks survive the continuous merging process in your model? Also, I did not understand how you assign $\mathrm{B} / \mathrm{D}$ ratios to your merger.

Roos: As a crude estimate I have assumed that disks are destroyed if the accreted mass is comparable to the disk mass. For smaller accreted masses I expect that accretion of smaller masses will produce thick disks. It seems likely that after such an accretion event the remaining gas in the disk will settle again in a thin disk. Regarding your second question, I just assumed that accretion of a smaller galaxy by a big disk galaxy will lead to the growth of the central bulge or spheroid.

Noreau: I have a big worry with your merging scheme from chemistry arguments. Smaller systems are usually metal poor. How can they contribute in building metal-rich bulges?

Roos: Small galaxies are more numerous, but the galaxies which contribute most to the mass of the central spheroid will have a mass which is comparable to the bulge mass. Such galaxies may not be so metal-poor. Nevertheless I do expect that many big bulges contain a population of metal-poor stars. Let me also make it clear that I did not consider the fate of the gas, not because I think it is not important, but because it is much more difficult to model. I merely want to point out that it may be possible to explain many aspects of the morphological evolution of galaxies in the simple-minded model I discussed.

Zasov: My question is related to the $B / D$ ratio and the luminosity modelling. The luminosity of a galaxy, especially a disk, is a very complex function which depends on the rate of evolution (which, in turn, may depend on $B / D$ ), internal absorption, etc. Isn't it more convenient to consider the velocity of rotation or, say, the halfwidth of the HI line? I suppose that if we take those instead of the luminosity, the difference between cluster and field galaxies may disappear.

Roos: I have assumed that the mass-to-light ratio is constant. It would indeed be interesting to know how $M / L$ varies with morphological type and/or with galaxy luminosity. 